OPEN ACCESS

Edited by:

Marco Tatullo,

Tecnologica S.r.l., Italy

Reviewed by:

Alessandra Pisciotta

University of Modena and Reggio

Emilia, Italy

Denitsa Docheva,

University Medical Center

Regensburg, Germany

${ }^{*}$ Correspondence: Lyndon F. Cooper

cooperlf@uic.edu

Specialty section:

This article was submitted to Craniofacial Biology and Dental

Research,

a section of the journal

Frontiers in Physiology

Received: 17 October 2019 Accepted: 13 December 2019

Published: 14 January 2020

Citation:

Cooper LF, Ravindran $S$ Huang C-C and Kang M (2020) A Role for Exosomes in Craniofacial Tissue Engineering and Regeneration.

Front. Physiol. 10:1569.

doi: 10.3389/fphys.2019.01569

\section{A Role for Exosomes in Craniofacial Tissue Engineering and Regeneration}

\author{
Lyndon F. Cooper*, Sriram Ravindran, Chun-Chieh Huang and Miya Kang \\ College of Dentistry, The University of Illinois at Chicago, Chicago, IL, United States
}

Tissue engineering and regenerative medicine utilize mesenchymal stem cells (MSCs) and their secretome in efforts to create or induce functional tissue replacement. Exosomes are specific extracellular vesicles (EVs) secreted by MSCs and other cells that carry informative cargo from the MSC to targeted cells that influence fundamental cellular processes including apoptosis, proliferation, migration, and lineage-specific differentiation. In this report, we review the current knowledge regarding MSC exosome biogenesis, cargo and function. This review summarizes the use of MSC exosomes to control or induce bone, cartilage, dentin, mucosa, and pulp tissue formation. The next-step engineering of exosomes provides additional avenues to enhance oral and craniofacial tissue engineering and regeneration.

Keywords: exosome, oral, tissue engineering, bone, dentin, cartilage

\section{INTRODUCTION}

The reconstruction of craniofacial tissues embraces a broad set of biological and clinical interests. Whether the particular biological or clinical concern involves bone, cartilage, salivary glands, skin and mucosa, muscle or nerve, the fundamental principles and challenges of tissue regeneration are shared. Classically, tissue engineering efforts directed toward tissue regeneration involve three key elements. Scaffolds, growth factors and cells are used either separately or together in efforts to replace functional tissues (Langer and Vacanti, 1993). Regarding the cellular component of tissue engineering, mesenchymal stem cells (MSCs) have been the focus of many efforts for craniofacial regeneration. MSCs are widely available from diverse tissues and are capable of expansion (limited self-renewal) and multi-lineage differentiation into bone, cartilage, fat, muscle, and nerve (Pittenger et al., 1999). They do undergo eventual senescence and require significant time for expansion in culture. The use of allogeneic cells requires intensive screening to preclude disease transmission. Yet, unlike the use of embryonic stem cells or induced pluripotent stem cells, MSCs do not pose the same risk of teratoma formation following transplantation or bear the same ethical issues associated with ESC usen (Kim and Park, 2017). While tumor formation and chromosomal abnormalities have been reported in mouse studies, MSC performance in large animal studies or early phase clinical trials have not revealed tumorigeneses or ectopic tissue formation (Williams and Hare, 2011).

Mesenchymal stem cells may function in several ways following transplantation. Transplanted MSCs may engraft and differentiate to directly form new tissues, they may mediate host cell formation of new tissues by paracrine signaling to induce differentiated cells to direct tissue regeneration and angiogenesis, or they may control wound healing and regeneration by immunomodulatory regulation involving direct cell-to-cell or indirect secretory signaling. 
Immunomodulation is recognized as an important aspect of MSC function in wound healing and thus tissue engineering. These mechanisms may not be mutually exclusive and, importantly, local cues may influence tissue specific/lineage specific functions of the MSC in tissue regeneration (Gnecchi et al., 2005; Phinney and Pittenger, 2017). However, the paracrine MSC function may profoundly influence the success of MSC-based tissue engineering by affecting host stem cell protection, apoptosis, neo-vascularization, and recruitment and differentiation (Caplan and Dennis, 2006; Gnecchi et al., 2008). This underscores the advantages of engrafted MSCs as a source of sustained release of factors in response to local conditions.

Despite the reported advantages of MSCs, the clinical use of MSCs face pragmatic and regulatory hurdles. Expanding autologous cells is time-intensive and does not permit real-time therapeutics and expansion may exceed "minimal manipulation" guidelines, it requires screening of donors for allogenic cells, there are challenges of biobanking and cryopreservation, and the concept of potency and dosing of MSCs for regenerative therapies remains to be clarified (Liang et al., 2014).

Earlier studies of myocardial regeneration demonstrated that the conditioned media of MSCs could support myocardial regeneration in vivo (Gnecchi et al., 2005). While conditioned media has traditionally been considered a source of cytokines and growth factors supporting regeneration or lineage-specific differentiation, Lai et al. (2010) demonstrated that the exosomes of MSC conditioned media also positively influenced cardiac tissue regeneration/repair. Since then, there has been an expanding interest in the use of MSC exosomes as a cellfree alternative to MSCs for the purpose of directing tissue regeneration and tissue engineering (Phinney and Pittenger, 2017). Exosomes may exhibit functions attributed to the MSC that influence different target cells and their functions by controlling proliferation, differentiation, migration and apoptosis (Figure 1). MSC exosomes may present similar advantages, opportunities, and challenges the context of craniofacial regeneration (Pilipchuk et al., 2015). This review will consider the role of exosomes in tissue engineering and their potential use in craniofacial regeneration and repair.

\section{EXOSOMES DEFINED}

Exosomes are specific extracellular vesicles (EVs) secreted into extracellular fluid (and culture media) by all cells. EVs include apoptotic bodies $(500 \mathrm{~nm}-2 \mu \mathrm{m})$, microvesicles $(100-1000 \mathrm{~nm})$ and exosomes (30-150 nm). Exosomes were identified by Pan and Johnstone (1983) and were distinguishable from other extracellular vesicles by their biogenesis, size and biochemical composition. Exosomes are 30-150 nm vesicles derived from inward budding of endosomal membranes of multi-vesicular endosome (MVE) to form intraluminal vesicles (ILV). Fusion of the MVE with the plasma membrane results in the release individual exosomes (Kowal et al., 2014). These nanoscale lipid bilayer exosomes carry lipids, mRNA, miRNA and proteins derived from the parental cell (Figure 2). The biogenesis of exosomes involves the sorting of lipid-membrane associated

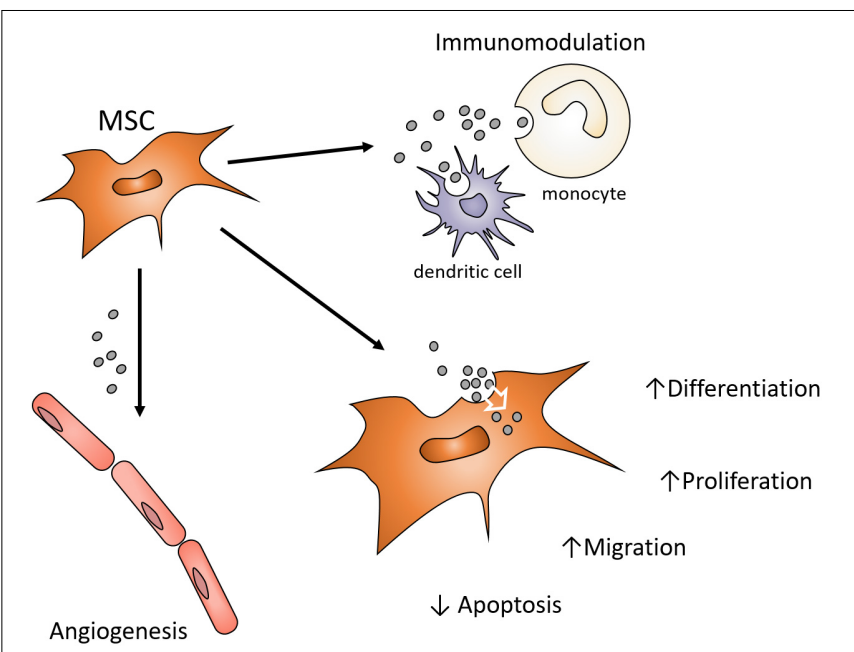

FIGURE 1 | Possible MSC exosome functions contributions to paracrine signaling in Bone Regeneration. MSC exosomes endocytosed by various local cells can influence angiogenesis, inflammation and the functions of osteoprogenitor cells. Enhancing functions of endogenous cell types involved in craniofacial regeneration avoids the harvesting, expansion and use of autogenous or allogenic cells. Exosomes can provide alternative modulation of cell function to avoid use of growth factors or cytokines in order to alter target cell function.

proteins by ESCRT-dependent and -independent mechanisms, intracellular exosome trafficking and the endocytosis of exosomes by recipient (target) cells and this has been comprehensively reviewed (van Niel et al., 2018).

Exosomes of all cells are characterized by exosome-specific protein and lipid content. The exosome lipid bilayer is specific to EVs with noted enrichment in cholesterol, sphingomyelin, phosphatidyl serine and notable similarity to lipid rafts (Tan et al., 2013; Skotland et al., 2019). Exosomes contain plasma, cytosolic and nuclear proteins (Kowal et al., 2014). Several proteins are associated with exosome biogenesis (ESCRT proteins, Alix and TSG 101) and membrane function (RAB proteins, annexins, integrins, tetraspanin, MHC class II, CAMs). HSP70 and HSP 90 are common cargo proteins (Kalluri, 2016). In a proteomic analysis of MC3T3-E1 cell exosomes, 1069 proteins were identified and 786 overlapped with the current ExoCarta database. The associated pathway analysis revealed Integrin and mTOR signaling pathways, both of which are important in osteoblast differentiation and bone formation (Ge et al., 2015). Differences in EV protein content of MSCs from different sources implies unique functionality of EV from these different sources (van Balkom et al., 2019). A recent substantial review of the proteomic content of MSC EVs revealed that despite the different isolation methods and MSC sources, $44 \%$ of proteins are common to 7 of 10 datasets of EV proteins versus only $20.4 \%$ common MSC proteins compared to EV of non-MSCs. MSC exosomes, as currently isolated, may represent a heterogeneous population of nanoscale EVs that contain different protein cargo (Toh et al., 2018a); further development of isolation techniques may improve the ability to direct protein cargo of exosomes for therapeutic use. Studies focused on the MSC exosome proteins 



FIGURE 2 | (A) Exososome biosynthesis. Exosomes are formed from endosomes (1) by an inward budding process to form intracellular vesicles (2). These mature as multivesicular bodies (3) that fuse with the cell plasma membrane to release exosomes. (B) Exosomes are 30-150 nm extracellular vesicles containing specific proteins, RNAs and lipids. Proteins include HSP70, 90, GAPDH; proteins involved in synthesis (Alix, ESCRT proteins, TGS101), and membrane associated or transmembrane proteins (RABs, Annexins, CAMs, Integrins, Tetraspanins, MHC I and II) and other cytosolic proteins.

indicate that MSC exosomes may deliver specific proteins to control aspects of regeneration that include apoptosis (Lai et al., 2013), angiogenesis (Anderson et al., 2016), cell migration (Zhang et al., 2015) and lineage-specific cell differentiation (Wu et al., 2018). Anderson et al. (2016) noted that the proteome of MSC exosomes lack signaling proteins such as cytokines and growth factors, but instead contain downstream mediators of these pathways. Exosomal proteins may compliment the soluble growth factor/signaling of MSCs, thereby constituting a "dualpath" paracrine signaling mechanism of the secretome.

Significant effort has been extended to define the exosomes' content ("cargo") that is a result of exosome biogenesis involving direct and indirect targeting of cellular constituents. Exosomes contain protein, mRNA, miRNAs, and lncRNAs. Perhaps the most abundant component of the exosome cargo is miRNAs, non-coding RNAs (17-24 nucleotides) that regulate gene expression. MSC exosomes contain approximately 150 miRNAs (Lai et al., 2015) encoding regulators of signaling pathways involved in repair and regeneration (e.g., ERK, SMAD). ExoCarta $\left.{ }^{1}\right)$ is an exosome database providing data and statistics regarding exosomal cargo including identified miRNAs. A second open resource for EVs is Vesiclepedia ${ }^{2}$.

Exosome miRNA content is representative of the parental cell and indicates that exosome miRNA content is selective and appears to be specific to the exosome derived cell type and cell condition (e.g., hypoxia, inflammation). Furthermore, it is possible to direct miRNAs into exosomes using a specific EXOmotif (GGAG) (Villarroya-Beltri et al., 2013). Thus, miRNAs can be engineered to therapeutically alter cell functions. Exosome

${ }^{1}$ ExoCarta.org

${ }^{2}$ microvesicles.org
miRNA cargo specificity may also be diagnostic. While less abundant, lncRNAs may be important as constituent exosomal cargo. IncRNA control of cell function in genetic reprograming implies that exosomal lncRNA may also function in exosomemediated changes in target cell function (Conigliaro et al., 2017). The exosome cargo appears to be a specifically encapsulated mixture of proteins and RNAs that present valuable new opportunities for diagnostics and therapeutics.

\section{EXOSOME FUNCTION IN PARACRINE SIGNALING; HORIZONTAL TRANSFER OF PROTEIN AND RNA}

Exosomes were initially considered carriers of insignificant cellular debris. The current study of exosomes as mediators of cell signaling was advanced by Raposo et al. (1996), Zitvogel et al. (1998), and Raposo and Stoorvogel (2013) who demonstrated that lymphocyte exosomes carried MHC class II antigens from cells to the extracellular fluid and induced a MHC class II T cell response, thereby suggesting that exosomes serve to convey signals from cell to cell. Subsequently, Valadi et al. (2007), showed that exosomes contained mRNA and miRNA that was shuttled by exosomes from one cell to another. Additional studies have demonstrated that miRNA transport to target cells by exosomes results in miRNA-mediated alteration in gene expression and cellular function. Importantly, exosomes mediate the horizontal transfer of mRNA from the parental cell to the target cell and imply an important paracrine signaling mechanism previously not understood (Tomasoni et al., 2013).

There exists substantial evidence that miRNA cargo participates in exosome-mediated paracrine signaling. Using 
Drosha knockout mice that have demonstrable inability to produce miRNAs, Collino et al., demonstrated that the MSCs of Drosha KO mice with Acute Kidney Injury and the MSCs depleted of Drosha were unable to activate regenerative gene expression in injured kidneys. Implied is that the exosome miRNAs function in the paracrine signaling of regeneration (Collino et al., 2015). Argonaut 2 (Ago2) binds to miRNAs to generate Ago2-miRNA complexes that are sorted to exosomes (McKenzie et al., 2016) and serves to cleave targeted mRNAs. The knockdown of Ago2 in MSCs rendered the MSC exosomes incapable of promoting cortical axonal growth compared with native MSC exosomes (Zhang et al., 2017). This study further demonstrated that miR-17-92 cluster-enriched exosomes enhanced axonal growth, demonstrating that modified miRNA exosome content alerted a specifically targeted cellular response. Indirectly, CD9-/- mice produce fewer exosomes and displayed diminished bone fracture healing (Furuta et al., 2016). These different studies all implicate exosome miRNA in mediating MSC exosome paracrine signaling.

The signaling by exosomes can serve regenerative therapy (Figure 2). Exosomes are able to alter cellular activities related to: epithelial/mesenchymal transition, matrix remodeling, apoptosis, cellular differentiation, inflammation and repair processes (Guo et al., 2017). To date exosome administration has been associated with the prevention of apoptosis, promotion of proliferation, directing differentiation and the promotion of neovascularization. Thus, exosome-mediated regeneration has become a topic of intense interest in several fields.

Recent reviews highlight the potential of exosomes to direct regeneration, to further manipulate exosome cargo and target exosomes to target cells and to formulate specific modes of delivery to damaged cardiac tissues (Shanmuganathan et al., 2018; Zamani et al., 2018). Bruno et al. (2009) who used MSC exosomes to reduce experimental kidney damage also revealed early evidence of exosome function in regeneration. Neurological and ophthalmological fields are active in the exploration of exosomes in regenerative therapy. In a rat model, intravitreal MSC exosome treatment preserved retinal ganglion cell function and promoted regeneration of axons. Importantly, the effects were mitigated by knockdown of Ago2, a central regulator of miRNA function, suggesting that MSC exosome effects are miRNA dependent (Mead and Tomarev, 2017).

The regeneration of other tissues is now being studied in the context of exosome function and therapeutics. For example, a role for specific exosomal miRNAs in nerve regeneration has demonstrated the favorable modulation of nerve injury (Chen et al., 2019). MSC exosomes may function as regulators of skeletal muscle regeneration. The administration of MSC exosomes to C2C12 cells induced MyoD and Myogenein gene expression and administration of MSC exosomes following cardiotoxininduced muscle injury resulted in enhanced muscle regeneration as demonstrated by greater diameter and numbers of myofibers, enhanced angiogenesis and reduced fibrosis (Nakamura et al., 2015). In studies involving nerve cell, macrophage, and MSC derived exosomes, all appear to influence inflammation, apoptosis, proliferative and differentiation/axonal outgrowth during peripheral nerve regeneration (Qing et al., 2018).
The control of angiogenesis is central to all regenerative and wound healing efforts (Park and Gerecht, 2014) and important observations have been made regarding the potential utility of MSC exosomes to promote angiogenesis. MSCs influence angiogenesis by secretion of paracrine factors (Watt et al., 2013); today exosomes are regarded as influential members of the MSC secretome. MSC exosomes may promote angiogenesis required for regenerative strategies affecting diverse target tissues. For example, MSC exosomes transfer miRNAs to HUVEC cells to promote tube formation and endothelial cell mobilization (Gong et al., 2017). However, MSC exosomes can exert both pro-and anti-angiogenic effects influencing VEGF expression. Further ROS and hypoxia influences on MSCs can alter the exosome cargo and exosome influence on angiogenesis (Alcayaga-Miranda et al., 2016). For these reasons perhaps, MSC-derived exosomes have been successfully used in numerous studies of regenerative medicine (Codispoti et al., 2018).

\section{MSC EXOSOME ROLES IN ORAL AND CRANIOFACIAL TISSUE ENGINEERING}

Exosomes harvested from MSCs derived from oral tissues may be isolated and used for craniofacial tissue engineering. Exosomes from diverse tissues may also be used to promote craniofacial repair and regeneration (Table 1). The potential use of exosomes for craniofacial and oral tissue regeneration embraces bone tissue engineering. MSC exosomes have be used to enhance fracture healing in mice. Importantly, when CD9-/mice that display reduced exosome production and display retarded callus formation were treated with exosomes, CD9-/mouse fracture healing was accelerated (Furuta et al., 2016). Mechanistic studies suggest that exosome miRNAs regulated osteoblastic differentiation. Specific pathways including Wnt pathway and PI3K/Akt pathways may be affected by MSC exosomes (Hao et al., 2017).

Several studies have demonstrated that exosomes influence MSC osteoinduction. Exosomes from differentiating osteoblastic cells (Cui et al., 2016), dendritic cells (Wang Z. et al., 2014), and monocytes (Ekstrom et al., 2013) can potentiate MSC osteoblastic differentiation in cell culture. MSC exosomes and exosomes of MSCs undergoing osteoblastic differentiation also induce osteoblastic differentiation. In a study involving mineralizing MC3T3-E1 cells, 43 highly expressed miRNAs that, upon gene ontology and pathway network analysis, revealed their possible action in Wnt, Insulin, TGFB, and calcium signaling pathways. Associated with increased target ST2 cell osteogenesis, mineralizing MC3T3-E1 cell exosomes inhibited Axin1 expression and increased $\beta$-catenin expression (Cui et al., 2016). Similarly, C2C12 cells expressed 12 miRNAs highly in isolated exosomes that promoted MC3T3-E1 cell osteoblast differentiation. When miR-27a-3p was depleted, targeted MC3T3-E1 cell exosome-dependent differentiation was prevented. A $\beta$-catenin dependent mechanism mediated this exosome (+miR-27a-3p) action in osteoblasts (Xu et al., 2018). MSC exosomes carry miRNAs that impact osteoblast physiology and bone regeneration. Several reviews have tabulated many 
TABLE 1 | MSC exosomes in craniofacial repair and regeneration.

\begin{tabular}{|c|c|c|c|c|}
\hline Tissue & Exosome source & References & Type of study & Observation \\
\hline \multirow[t]{6}{*}{ Bone } & Bone marrow MSC & Cui et al., 2016 & In vitro & $\begin{array}{l}\text { Mineralizing osteoblast exosomes induce } \\
\text { osteogenic differentiation of precursors }\end{array}$ \\
\hline & & Narayanan et al., 2016 & In vitro & $\begin{array}{l}\text { Differentiating MSC exosomes induce osteogenic } \\
\text { differentiation of naïve MSCs }\end{array}$ \\
\hline & Dendritic cells & Wang Z. et al., 2014 & In vitro & $\begin{array}{l}\text { Dendritic cell exosomes trigger MSC osteogenic } \\
\text { differentiation }\end{array}$ \\
\hline & Adipose MSC & Li W. et al., 2018 & $\begin{array}{l}\text { In vivo, mouse calvarial } \\
\text { defect model }\end{array}$ & $\begin{array}{l}\text { Tissue engineered bone graft doped with } \\
\text { exosomes induces bone regeneration }\end{array}$ \\
\hline & & Lu et al., 2017 & In vitro & $\begin{array}{l}\text { TNF } \alpha \text {-preconditioned MSC exosomes induce } \\
\text { osteogenic differentiation of naïve MSCs }\end{array}$ \\
\hline & Monocytes & Ekstrom et al., 2013 & In vitro & $\begin{array}{l}\text { Monocyte-derived exosomes promote osteogenic } \\
\text { gene expression in MSCs }\end{array}$ \\
\hline \multirow[t]{3}{*}{ Cartilage } & Bone marrow MSC & Zhang S. et al., 2018 & $\begin{array}{l}\text { In vivo, rat osteochondral } \\
\text { defect model }\end{array}$ & $\begin{array}{l}\text { MSC exosomes promote repair by attenuating } \\
\text { apoptosis, enhancing proliferation and reducing } \\
\text { immune reactivity }\end{array}$ \\
\hline & Embryonic MSC & Zhang S. et al., 2016 & $\begin{array}{l}\text { In vivo, rat osteochondral } \\
\text { defect model }\end{array}$ & $\begin{array}{l}\text { MSC exosomes promote healing of rat trochlear } \\
\text { groove osteochondral defect }\end{array}$ \\
\hline & Chondrocytes & Chen et al., 2018 & $\begin{array}{l}\text { In vivo, rabbit } \\
\text { progenitors }+ \text { alginate } \\
\text { scaffolds in nude mice }\end{array}$ & $\begin{array}{l}\text { Chondrocyte exosomes promote subcutaneous } \\
\text { stable ectopic chondrogenesis }\end{array}$ \\
\hline Dentin/dental pulp & DPSC & Huang et al., 2016 & $\begin{array}{l}\text { In vivo, subcutaneous root } \\
\text { slice model }\end{array}$ & $\begin{array}{l}\text { Exosomes from differentiating DPSCs promote } \\
\text { dentin/pulp regeneration in a subcutaneous tooth } \\
\text { root slice regeneration model }\end{array}$ \\
\hline \multirow[t]{2}{*}{ Periodontal ligament } & Bone Marrow MSC & Chew et al., 2019 & $\begin{array}{l}\text { In vivo, rat periodontal } \\
\text { defect model }\end{array}$ & $\begin{array}{l}\text { MSC exosomes improve periodontal ligament } \\
\text { function and promote regeneration }\end{array}$ \\
\hline & Adipose MSC & Mohammed et al., 2018 & In vivo, rat ligature model & $\begin{array}{l}\text { Adipose MSC exosomes can be used as a } \\
\text { non-surgical adjunct treatment to improve } \\
\text { periodontal repair and regeneration }\end{array}$ \\
\hline
\end{tabular}

miRNAs (primarily derived from osteoblastic cell lines and MSCs) presently implicated in control of osteoblast function and related bone regeneration (Papaioannou et al., 2014; Fang et al., 2015; Huang et al., 2017). In aggregate, these reviews demonstrate that MSC exosomes carry many miRNAs that impact key signaling pathways that include TGF $\beta$, BMP, and Wnt pathways to influence SATB2, Runx2, Dlx5, and Osx function in control of osteoblast differentiation. Such studies indicate that exosomes modulate the physiology diverse target cells within regenerating bone tissue to influence the process of bone regeneration and repair.

The osteoinductive potential of MSC exosomes is apparently dependent upon the state of the parental cell. When human Adipose-derived MSC exosomes were obtained following osteogenic induction, the exosomes have greater osteoinductive effect. These exosomes accelerated bone healing in a calvaria defect model (Li W. et al., 2018). By pretreatment of Adiposederived MSC with TNF $\alpha$ to mimic the acute inflammatory phase of wound healing, proliferation, migration, osteogenic gene expression and mineralization was increased in target hOB cell culture. This was attributed, in part, to increased Wnt3 within exosomes purified from TNF $\alpha$ treated hOB (Lu et al., 2017).

Exosomes from human iPS-MSC exosomes promoted osteoblast proliferation and differentiation in cell culture and, when applied with a $\beta$ TCP scaffold for healing of rat calvaria critical-sized bone defects, the exosomes enhanced bone regeneration and angiogenesis (Qi et al., 2016). In addition, using human iPS-MSC exosomes, a dose-dependent increase in rat calvaria critical-sized defect bone regeneration was demonstrated by $\mu \mathrm{CT}$ and immunohistochemistry. In parallel, gene expression analysis implicated the activation of the PI3K/Akt pathway in iPS-MSC exosome treated BMSCs and this was affirmed using the Pi3K inhibitor LY294002 to block the effect of exosomes on osteogenesis (Zhang J. et al., 2016).

Cartilage repair and regeneration is affected by MSC exosomes and those of chondrocytes. The treatment of rat osteochondral defects with exosomes isolated from human MSCs (derived from HuES9 human embryonic stem cells) induced complete cartilage and subchondral bone repair at 12 weeks that was equivalent to unoperated control sites (Zhang S. et al., 2016). The authors indicated that many components of the MSC exosome were required for effective tissue regeneration and further suggested that exosomes represented an advantageous "cell-free" strategy for leveraging human embryonic MSCs for cartilage repair. Toh et al. (2017) reviewed the potential use of exosomes for induction of chondrogenic differentiation and upregulation of chondrogenic transcription factors (Toh et al., 2017). Further, it was demonstrated that MSC exosome-mediated cartilage repair also involved the specific immune modulation as demonstrated by the selective alteration of the local population of regenerative M2 macrophages versus pro-inflammatory M1 macrophages (Zhang S. et al., 2018). The potential to tailor exosomemediated regenerative therapies to unique clinical conditions was recently demonstrated (Tao et al., 2017). They engineered human 
synovial MSC exosomes to carry increased levels of miR-140-5p with the successful purpose of directly increasing proliferation and migration of targeted chondrocytes without suppression of ECM protein synthesis. MSC exosomes may exert specific influences on different cells within the local environment to control regeneration.

When exosomes purified from human MSC conditioned media were applied weekly to healing of critical sized osteochondral defects in the distal femurs of Sprague Dawley rats, increased ICRS scores and O'Driscoll scores were notes with histological evidence of increased tissue repair. Exosome treatment over 12 weeks achieved regeneration hyaline cartilage and subchondral bone compared to fibrous repair in the PBS treated control limbs. The effect observed was attributed to multiple functions of exosomes including immunomodulation and an array of exosomal components required to orchestrate tissue regeneration of bone and cartilage (Zhang S. et al., 2016).

Additionally, chondrocyte-derived exosomes directed the experimental subcutaneous formation of cartilage-like tissues. It was suggested that maintaining a favorable chondrogenic "niche" was the result of antiangiogenic factors of chondrogenic exosomes (Chen et al., 2018). Many exosomes possess immunomodulatory function that is essential to the process of cartilage regeneration (Toh et al., 2017). This was reflected in a recent report of M2 macrophage polarization in healing osteochondral defects following MSC exosome treatment. In this study, increased cell proliferation, reduced apoptosis and enhanced matrix synthesis accompanied cartilage repair and regeneration (Zhang S. et al., 2018). These studies of cartilage repair indicate that exosomes are effective in directing tissuespecific regeneration that involves the targeting of multiple biological and cellular processes.

Exosome function is well defined in development (Hayashi and Hoffman, 2017) and has been convincingly demonstrated by example in the submandibular gland. Exosomal miRNAs transferred from mesenchymal cells to epithelial progenitors experience miRNA dependent epigenetic regulation of gene expression. Exosomes also function during reciprocal epithelium-mesenchyme interactions during tooth development. When Rab27a/b knockdown was used to disrupt exosome biosynthesis, enamel and dentin formation was reduced in organ culture experiments. Accordingly, the investigators further identified miR135a as an exosome component influencing Wnt signaling that increased dentin matrix protein expression (Jiang et al., 2017). Another recent study demonstrated exosome miRNA regulation of apoptosis in tooth development where the upregulation of miR133b - induced apoptosis in primary dentin mesenchyme and its ectopic expression prevented proper experimental tooth formation (Li Y. et al., 2018). Such studies implicate exosomes as key signaling vehicles in oral and craniofacial tissue development and suggest that exosomes may be utilized in their regeneration by control of apoptosis, proliferation, and differentiation of targeted cells.

Despite advances using exosomes in other fields of medicine, even the most recent general reviews of periodontal tissue regeneration have not considered that MSC exosomes have the potential to direct periodontal regeneration and repair (Chew et al., 2019). Adipose derived stem cell exosomes used to augment non-surgical periodontal therapy in a rat model of ligature-induced periodontitis positively affected periodontium regeneration (Mohammed et al., 2018).

\section{A ROLE FOR MSC EXOSOMES IN IMMUNOMODULATION}

Mesenchymal stem cells are widely distributed among tissues and appear to communicate with the local inflammatory microenvironments in the process of tissue repair and regeneration. It has been suggested that MSCs are involved in the recruitment and modulation of inflammatory cells during repair and regeneration. Following damage, recruited MSCs are known to produce immunoregulatory factors that contribute to the progression of inflammation and wound healing. The MSC secretome is known to contain many growth factors and cytokines that direct angiogenesis, extracellular matrix synthesis and remodeling as well as progenitor cell differentiation. With regard to immunomodulation, MSCs act by direct cell contact with immune cells and they secrete potent factors including TGF- $\beta$, NO, PGE2, 1L-17, and IL-10. MSCs communicate to alter TC, dendritic cell, B cell and NK cell activities to modulate inflammation during the repair process. While the immunomodulatory role of MSCs has been broadly reviewed (Wang Y. et al., 2014; Gao et al., 2016), a role for exosomes as part of the MSC's immunomodulatory secretome has yet to be fully characterized.

MSC exosomes have been examined in the context of immunomodulation. The potential mechanisms of immunomodulation by MSC exosomes include their impact on tissue resident macrophages that includes suppression of M1 macrophage polarization and enhancement of M2 macrophage activation. MSC exosomes achieve this alteration of M1/M2 ratios in healing tissues by directly targeting signaling pathways influencing macrophage polarization. Beyond the macrophage, MSC exosomes also influence dendritic cells, B cells, and NK cells of the immune system. The prominent effect of MSCs on $\mathrm{T}$ cell function is well known and several reports indicate that MSC exosomes can modulate T cell function (Seo et al., 2019). It has been noted that tissue macrophages may vary in their influence on immunomodulation. It remains to be determined if exosomes of MSCs from various craniofacial tissues (e.g., gingiva versus dental pulp vs. alveolar bone) possess unique immunomodulatory functions.

When MSC exosomes have been studied in therapeutic animal models of cardiac infarction, liver disease, ischemic stroke brain injury, or induced kidney disease, the investigators have reported altered or reduced immune cell invasion into sites of injury or a decline in pro-inflammatory reactions (Borger et al., 2017). MSC exosomes appear capable of modulating both humoral and cellular immune responses (Toh et al., 2018b). Several clinical trials currently underway involve MSC exosomes for the treatment of type I diabetes, for chronic kidney disease, for macular degeneration and for ischemic stroke (Mendt et al., 2019). It is feasible that MSC exosomes can be 
applied clinically for local control of inflammation to enhance craniofacial regeneration or suppress disease.

The possible advantages for using MSC exosomes for immunomodulation include overcoming disadvantages of using cell-based therapies (e.g., variability in cell isolation and expansion, dosing, targeting) as well as being able to produce large numbers of exosomes from highly characterized parental cells or cell lines. In a translational effort, Toh and colleagues recently demonstrated that human MSC exosomes enhanced periodontal ligament cell migration and modestly enhanced periodontal regeneration as assessed by $\mu \mathrm{CT}$ and histological methods (Chew et al., 2019). In a similar manner, MSC exosomes enhanced repair of osteochondral defects that was associated with a reduction in proinflammatory cytokines and increased numbers of regenerative (M2) macrophages (Zhang S. et al., 2018).

\section{EXOSOME ISOLATION FROM DENTAL TISSUE}

Exosomes may be isolated from MSCs of dental tissues (Figure 3). Either exfoliating deciduous teeth or intentionally harvested adult teeth can provide dental pulp stem cells (DPSCs) for regenerative purposes (Gronthos et al., 2000). DPSCs direct regeneration by in vivo multipotential differentiation, including liver, dental pulp, bone, muscle and nerve, besides promoting vascularization of the regenerating tissues (Gandia et al., 2008; Ikeda et al., 2008; Yamada et al., 2011; Bianchi et al., 2017; Zordani et al., 2019). DPSCs of neural crest derivation may provide particularly valuable function in nerve regeneration as demonstrated by their phentoype (Carnevale et al., 2018), their differentiation along neurogenic lineages and functional studies demonstrating DPSC-mediated functional regeneration of siatic nerve (Ullah et al., 2017). A primary limitation of DPSCs is the relatively small number of cells obtained for autologous use in regenerative medicine (Tatullo et al., 2015), yet they have been advocated as a suitable MSC for tissue/cell banking (Lizier et al., 2012). A primary advantage of the DPSC versus the bone marrow derived MSC is the relative availability and ease of access (extraction/endodontics). Our efforts to explore DPSC exosomes may provide an allogenic resource for regeneration using culture expanded or immortalized DPSCs. In light of the long-term stability of exosomes (Kalra et al., 2013; Lee et al., 2016) pulp derived exosomes may play a significant role in regenerative medicine. We and others, in preliminary cell culture and animal studies have affirmed that DPSC exosomes, like MSC exosomes promote immune-protective and anti-apoptotic mechanism in targeted cells and tissues (Jarmalaviciute et al., 2015) and, more specifically, DPSC exosomes demonstrate pulp regeneration potential (Huang et al., 2016).

Periodontal ligament cells have been an historical target source of multipotential stem cells to direct regeneration (Seo et al., 2004). Periodontal ligament stem cells (PDLSCs) have been isolated from the periodontal ligament (Zhu and Liang, 2015). PDLSCs differentiate along osteogenic, adipogenic, and chondrogenic lineages (Gay et al., 2007). Additionally, they are self-renewing when transplanted into ectopic sites and can differentiate to form mineralized structures in vivo (Menicanin et al., 2014). Although the regenerative properties of PDLSCs has been studied extensively, the PDLSC derived exosomes have not been studied as extensively as the bone marrow derived MSCs (BMSC) exosomes.
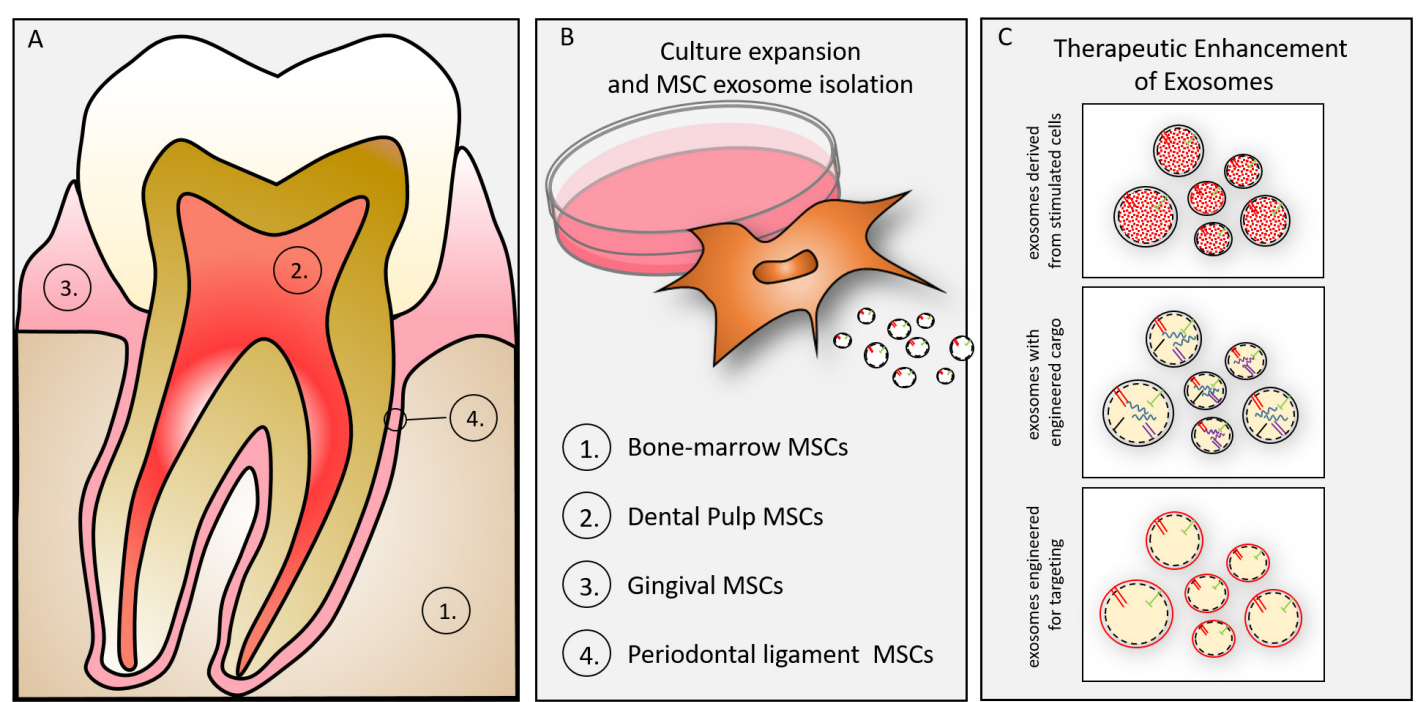

FIGURE 3 | (A) Oral tissue-specific cell types are sources of unique exosomes. Exosomes have been isolated from cells of (1) bone, (2) dentin/pulp, (3) gingiva, and (4) periodontal ligament. These cell-type specific exosomes may provide lineage specific direction to craniofacial tissue engineering. (B) Culture expansion and MSC exosome isolation. MSCs can be isolated from many oral/craniofacial tissues and expanded in culture. MSCs have been isolated from bone marrow, dental pulp, gingiva and periodontal ligament. (C) MSC derived exosomes can be enhanced for therapeutic advantage by several techniques including (1) parental cell stimulation (e.g., growth factor, culture conditions), (2) engineering to direct inclusion of overexpressed miRNAs, (3) engineering to include membrane proteins (e.g., integrins) to target exosomes to tissues (or cells). 
The documented immunomodulatory properties of PDLSCs (Wada et al., 2009) have been attributed to paracrine function (Rajan et al., 2016b) and this is likely mediated by PDLSC derived exosomes (Rajan et al., 2016a; Misawa et al., 2019). Furthermore, exposure of PDLSCs to lipopolysaccharide triggers the production of exosomes that have the potential to induce proinflammatory M1 polarization (Kang et al., 2018). The similarity of PDLSCs with the bone marrow derived MSCs in terms of basic characteristics raises interesting questions about their potential as a source of therapeutic exosomes in regenerative medicine applications.

Exosomes have also been isolated from gingival MSCs and their administration to tongue wounds increased BDNF expression and associated taste bud regeneration with neurofilament expression reflecting re-innervation (Zhang Y. et al., 2018). Gingival MSCs isolated from gingival lamina propria were used in a diabetes-induced wound closure assay to facilitated recalcitrant healing. When gingival MSCs in a chitosan/silk hydrogel were applied to cutaneous wounds in a rat model, the addition of gingival MSC exosomes enhanced wound closure rates. Both nerve density and microvessel density were increased in gingival MSC exosome treated wounds (Shi et al., 2017).

\section{ENGINEERING EXOSOMES FOR THERAPEUTICS}

The innate characteristics of exosomes suggest innovative uses in clinical therapy. Their nanoscale size, membrane composition related to rapid endocytosis, relative lack of toxicity, stability, and access to the central nervous system (passing the blood brain barrier) combine to provide a vehicle for delivery of specific signals to target cells. These signals (protein, RNA, miRNA) represent lineage and physiology of the parental cell. Exosomes may also possess allogenicity enabling the use of well characterized and standardized donor sources. In addition, Xenographic success using human MSC exosomes to achieve positive results in animal studies has been repeatedly referenced in this review. However, the reciprocal xenograft studies have not been performed in vitro or in vivo and represent knowledge gaps that remain poorly understood.

Further, exosomes can be engineered to carry specified cargo and synthetic (pharmacologic) cargo for therapeutic intent (Arenaccio et al., 2019). Stimulation of, or the genetic manipulation of, parental cells can alter exosome cargo to enhance regeneration. Alternatively, selected siRNA, miRNA, drugs and enzymes can be loaded into exosomes directly by electroporation, chemical-based transfection, simple incubation methods (Johnsen et al., 2014; Liao et al., 2018). Early investigations also suggest that exosomes may be stored longer term, perhaps by lyophilization (Charoenviriyakul et al., 2018). Further engineering of exosomes can involve expression of surface proteins to direct exosomes to specific extracellular matrices or to selected cells (Figure 4).

Systemic administration of exosomes is challenged by the preferential uptake in liver, kidney and spleen or off target effects.
Targeting of exosomes to cells or tissues may be accomplished by expression of proteins on the lipid bilayer (e.g., specific integrin, viral glycoprotein). For example, exosomes from parental cells engineered to an EGFR-specific peptide ligand were successfully targeted to xenograft $\mathrm{EGFR}^{+}$breast cancer cells in mice (Ohno et al., 2013). Endogenous exosomal tetraspanin proteins may contribute the process of targeting. Targeting of exosomes was achieved by transfection of defined tetaspanin-integrins and intravenous injection of the engineered exosome resulted in preferential exosome uptake in the pancreas (Rana et al., 2012). Exosomal integrin involvement in exosome targeting was elegantly indicated by studies of exosomal localization in cancers where unique exosomal integrins interact with specific cell associated extracellular matrix (Hoshino et al., 2015). To deliver siRNA in exosomes specifically to the brain, the central nervous system-specific rabies viral glycoprotein (RVG) was cloned in the integral exosome LAMP2b protein. The RVG engineered exosomes were specifically taken up by Neur02A cells. Following systemic injection of GAPDH siRNAs containing RVG exosomes in ice, significant brain tissue specific reduction in GAPDH mRNA expression was noted compared to other tissues of naked siRNA injection (Alvarez-Erviti et al., 2011).

These early investigations have yet to fully explore some of the challenges facing the use of exosomes as therapeutic agents in craniofacial regeneration. First and foremost, isolation methods are yet fully reproducible and scalable to meet expectations of good clinical practice. This embraces the matter of quality assessment of consistent isolation and integrity. Second, exosome stability and shelf life must be further explored; recent evidence suggests that lyophilization is a potential solution (Bosch et al., 2016; Charoenviriyakul et al., 2018). Third, delivery of exosomes requires careful consideration regarding targeting of specific cells and tissues, their use systemically and potential off-target complications. Delivery and targeting may be enhanced by engineering of the exosomes. Finally, the dosing (concentration and timing) and potency of exosomes must be carefully considered and methods of defining these clinical parameters must be addressed and adopted.

\section{EXOSOME ROLE IN ORAL AND SYSTEMIC DISEASE DIAGNOSIS}

The role of salivary exosomes in diagnosing systemic and oral diseases is one example of current speculative applications for exosomes in medicine and dentistry (Han et al., 2018). The importance of saliva in the diagnosis of oral and systemic diseases is of growing interest. The field has expanded to use liquid biopsy (non-invasive biofluid tests) for analyses by proteomics, transcriptomics, microbiomics, metabolomics, and microRNAs. While not the intended focus of this review, exosome use in diagnosis of disease is of growing interest in medicine and dentistry and merits brief consideration here. Exosomes isolated from biofluids can overcome limitations such as relative low expression of biomarkers and degradation of RNAs and miRNAs in whole and glandular saliva. Exosomes can be isolated using commercially available kits, ultracentrifugation methods, 


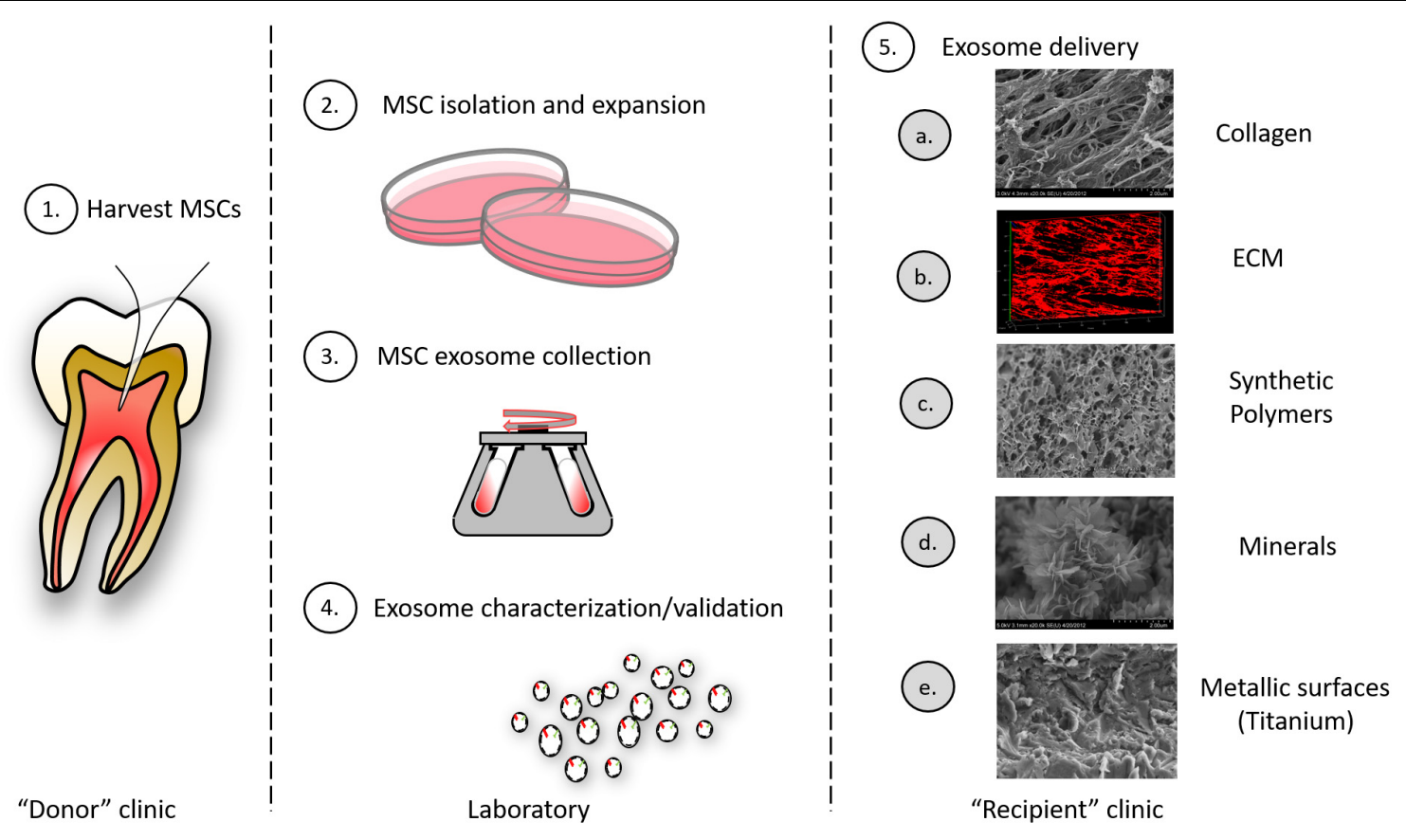

FIGURE 4 | Exosomes as therapeutic agents: exosomes may be clinically isolated from MSC isolated oral tissues (e.g., perhaps by pulpectomy). (1) This could be an autogenous scheme or, alternatively, MSCs could be isolated from pulp of extracted teeth and derived exosomes used in an allogenic scheme. (2) MSCs must be isolated and expanded and conditioned media from cells collected. (3) MSC exosomes are collected from conditioned media by different methods including ultracentrifugation, immune-isolation, and precipitation. (4) Exosomes must be characterized according to their size and integral membrane protein composition; engineered exosome cargo must be defined by miRNA or protein analysis. (5) Exosomes can be delivered to a variety of different tissues using different carriers that include: (a) collagen matrices, (b) decellularized ECM, (c) synthetic polymers (bioprinted carriers), (d) mineral scaffolds, and (e) metallic implants.

and specialized aqueous two-phase system to improve salivary exosome isolation (Sun et al., 2016; Kim et al., 2017). Exosomes isolated from glandular saliva were shown to possess miRNAs unique to salivary gland disease status (Michael et al., 2010). The use of salivary exosomes as biomarkers for cancer has been recently reviewed (Nonaka and Wong, 2017). Characterization of intact exosomes by FTIR distinguished exosomes of Oral Cancer subjects from Healthy individuals and suggests a noninvasive method for future diagnosis of oral cancer in early stages (Zlotogorski-Hurvitz et al., 2019). Subsequent studies oral cancer (Economopoulou et al., 2017; Lousada-Fernandez et al., 2018), oral lichen planus (Byun et al., 2015), and Sjogren's syndrome indicate the potential future use of salivary exosomes as diagnostic targets in liquid biopsy of oral and systemic disease.

\section{SUMMARY}

The past decade's exploration of exosome biology has extended to consideration of exosome's utility in controlling cell and tissue function during wound healing and regeneration. Significant progress has been made in understanding how exosome cargo is transferred to target cells and act as signals to alter cell function by means of packaged miRNAs and proteins. More recent advances in the engineering of exosomes demonstrate the possibility of generating cell free, nanoscale particles that can modulate specific cell functions and may target specific tissues and cells. The existing demonstration that MSC exosomes mediate the regeneration of bone, cartilage, dentin, muscle, blood vessels and nerves indicates that exosomes have possible application in oral/craniofacial regeneration. The role of MSC exosomes in immunomodulation is an important functionality that may have great importance in regeneration (and managing pathology) in the oral cavity. Given the stated advantages of exosomes when compared to other cell and molecular approaches to regeneration, exosome-based therapeutics may be of current importance in advancing oral/craniofacial regenerative therapy success.

\section{AUTHOR CONTRIBUTIONS}

LC conceived and performed the primary literature search of the document, and contributed to the writing and illustration of the manuscript. SR contributed to the writing of the document and illustrations. $\mathrm{MK}$ and $\mathrm{C}-\mathrm{CH}$ contributed to the literature search and writing of the document.

\section{FUNDING}

This study was supported by the NIDCR to SR (DE027404) and the NIH funded UIC Center for Clinical and Translational Studies to LC (UL1TR002733), for studies of exosomes in tissue engineering and immunomodulation, respectively. 


\section{REFERENCES}

Alcayaga-Miranda, F., Varas-Godoy, M., and Khoury, M. (2016). Harnessing the angiogenic potential of stem cell-derived exosomes for vascular regeneration. Stem Cells Int. 2016:3409169. doi: 10.1155/2016/3409169

Alvarez-Erviti, L., Seow, Y., Yin, H., Betts, C., Lakhal, S., and Wood, M. J. (2011). Delivery of sirna to the mouse brain by systemic injection of targeted exosomes. Nat. Biotechnol. 29, 341-345. doi: 10.1038/nbt.1807

Anderson, J. D., Johansson, H. J., Graham, C. S., Vesterlund, M., Pham, M. T., Bramlett, C. S., et al. (2016). Comprehensive proteomic analysis of mesenchymal stem cell exosomes reveals modulation of angiogenesis via nuclear factor-kappab signaling. Stem Cells 34, 601-613. doi: 10.1002/stem. 2298

Arenaccio, C., Chiozzini, C., Ferrantelli, F., Leone, P., Olivetta, E., and Federico, M. (2019). Exosomes in therapy: engineering, pharmacokinetics and future applications. Curr. Drug Targets 20, 87-95. doi: 10.2174/ 1389450119666180521100409

Bianchi, M., Pisciotta, A., Bertoni, L., Berni, M., Gambardella, A., Visani, A., et al. (2017). Osteogenic differentiation of hdpscs on biogenic bone apatite thin films. Stem Cells Int. 2017:3579283. doi: 10.1155/2017/3579283

Borger, V., Bremer, M., Ferrer-Tur, R., Gockeln, L., Stambouli, O., Becic, A., et al. (2017). Mesenchymal stem/stromal cell-derived extracellular vesicles and their potential as novel immunomodulatory therapeutic agents. Int. J. Mol. Sci. 18:E1450. doi: 10.3390/ijms18071450

Bosch, S., de Beaurepaire, L., Allard, M., Mosser, M., Heichette, C., Chretien, D., et al. (2016). Trehalose prevents aggregation of exosomes and cryodamage. Sci. Rep. 6:36162. doi: 10.1038/srep36162

Bruno, S., Grange, C., Deregibus, M. C., Calogero, R. A., Saviozzi, S., Collino, F., et al. (2009). Mesenchymal stem cell-derived microvesicles protect against acute tubular injury. J. Am. Soc. Nephrol. 20, 1053-1067. doi: 10.1681/ASN. 2008070798

Byun, J. S., Hong, S. H., Choi, J. K., Jung, J. K., and Lee, H. J. (2015). Diagnostic profiling of salivary exosomal micrornas in oral lichen planus patients. Oral Dis. 21, 987-993. doi: 10.1111/odi.12374

Caplan, A. I., and Dennis, J. E. (2006). Mesenchymal stem cells as trophic mediators. Journal Cell. Biochem. 98, 1076-1084.

Carnevale, G., Pisciotta, A., Riccio, M., Bertoni, L., De Biasi, S., Gibellini, L., et al. (2018). Human dental pulp stem cells expressing stro-1, c-kit and cd34 markers in peripheral nerve regeneration. J. Tissue Eng. Regen. Med. 12, e774-e785. doi: $10.1002 /$ term. 2378

Charoenviriyakul, C., Takahashi, Y., Nishikawa, M., and Takakura, Y. (2018). Preservation of exosomes at room temperature using lyophilization. Int. J. Pharm. 553, 1-7. doi: 10.1016/j.ijpharm.2018. 10.032

Chen, J., Ren, S., Duscher, D., Kang, Y., Liu, Y., Wang, C., et al. (2019). Exosomes from human adipose-derived stem cells promote sciatic nerve regeneration via optimizing Schwann cell function. J. Cell.Physiol. 234, 23097-23110. doi: $10.1002 /$ jcp. 28873

Chen, Y., Xue, K., Zhang, X., Zheng, Z., and Liu, K. (2018). Exosomes derived from mature chondrocytes facilitate subcutaneous stable ectopic chondrogenesis of cartilage progenitor cells. Stem Cell Res. Ther. 9:318. doi: 10.1186/s13287-0181047-2

Chew, J. R. J., Chuah, S. J., Teo, K. Y. W., Zhang, S., Lai, R. C., Fu, J. H., et al. (2019). Mesenchymal stem cell exosomes enhance periodontal ligament cell functions and promote periodontal regeneration. Acta Biomater. 89, 252-264. doi: 10.1016/j.actbio.2019.03.021

Codispoti, B., Marrelli, M., Paduano, F., and Tatullo, M. (2018). Nanometric biobanked msc-derived exosome (nanobiome) as a novel approach to regenerative medicine. J. Clin. Med. 7:357. doi: 10.3390/jcm7100357

Collino, F., Bruno, S., Incarnato, D., Dettori, D., Neri, F., Provero, P., et al. (2015). Aki recovery induced by mesenchymal stromal cell-derived extracellular vesicles carrying micrornas. J. Am. Soc. Nephrol. 26, 2349-2360. doi: 10.1681/ ASN.2014070710

Conigliaro, A., Fontana, S., Raimondo, S., and Alessandro, R. (2017). Exosomes: nanocarriers of biological messages. Adv. Exp. Med. Biol. 998, 23-43. doi: 10. 1007/978-981-10-4397-0_2

Cui, Y., Luan, J., Li, H., Zhou, X., and Han, J. (2016). Exosomes derived from mineralizing osteoblasts promote st 2 cell osteogenic differentiation by alteration of microrna expression. FEBS Lett. 590, 185-192. doi: 10.1002/18733468.12024
Economopoulou, P., Kotsantis, I., Kyrodimos, E., Lianidou, E. S., and Psyrri, A. (2017). Liquid biopsy: an emerging prognostic and predictive tool in head and neck squamous cell carcinoma (hnscc). Focus on circulating tumor cells (ctcs). Oral Oncol. 74, 83-89. doi: 10.1016/j.oraloncology.2017.09.012

Ekstrom, K., Omar, O., Graneli, C., Wang, X., Vazirisani, F., and Thomsen, P. (2013). Monocyte exosomes stimulate the osteogenic gene expression of mesenchymal stem cells. PLoS One 8:e75227. doi: 10.1371/journal.pone. 0075227

Fang, S., Deng, Y., Gu, P., and Fan, X. (2015). Micrornas regulate bone development and regeneration. Int. J. Mol. Sci. 16, 8227-8253.

Furuta, T., Miyaki, S., Ishitobi, H., Ogura, T., Kato, Y., Kamei, N., et al. (2016). Mesenchymal stem cell-derived exosomes promote fracture healing in a mouse model. Stem Cells Transl. Med. 5, 1620-1630.

Gandia, C., Arminan, A., Garcia-Verdugo, J. M., Lledo, E., Ruiz, A., Minana, M. D., et al. (2008). Human dental pulp stem cells improve left ventricular function, induce angiogenesis, and reduce infarct size in rats with acute myocardial infarction. Stem Cells 26, 638-645.

Gao, F., Chiu, S. M., Motan, D. A., Zhang, Z., Chen, L., Ji, H. L., et al. (2016). Mesenchymal stem cells and immunomodulation: current status and future prospects. Cell Death Dis. 7:e2062. doi: 10.1038/cddis.2015.327

Gay, I. C., Chen, S., and MacDougall, M. (2007). Isolation and characterization of multipotent human periodontal ligament stem cells. Orthod. Craniofac. Res. 10, 149-160.

Ge, M., Ke, R., Cai, T., Yang, J., and Mu, X. (2015). Identification and proteomic analysis of osteoblast-derived exosomes. Biochem. Biophys. Res. Commun. 467, 27-32. doi: 10.1016/j.bbrc.2015.09.135

Gnecchi, M., He, H., Liang, O. D., Melo, L. G., Morello, F., Mu, H., et al. (2005). Paracrine action accounts for marked protection of ischemic heart by akt-modified mesenchymal stem cells. Nat. Med. 11, 367-368.

Gnecchi, M., Zhang, Z., Ni, A., and Dzau, V. J. (2008). Paracrine mechanisms in adult stem cell signaling and therapy. Circ. Res. 103, 1204-1219. doi: 10.1161/ CIRCRESAHA.108.176826

Gong, M., Yu, B., Wang, J., Wang, Y., Liu, M., Paul, C., et al. (2017). Mesenchymal stem cells release exosomes that transfer mirnas to endothelial cells and promote angiogenesis. Oncotarget 8, 45200-45212. doi: 10.18632/oncotarget. 16778

Gronthos, S., Mankani, M., Brahim, J., Robey, P. G., and Shi, S. (2000). Postnatal human dental pulp stem cells (dpscs) in vitro and in vivo. Proc. Natl. Acad. Sci. U.S.A. 97, 13625-13630.

Guo, S. C., Tao, S. C., Yin, W. J., Qi, X., Yuan, T., and Zhang, C. Q. (2017). Exosomes derived from platelet-rich plasma promote the re-epithelization of chronic cutaneous wounds via activation of yap in a diabetic rat model. Theranostics 7, 81-96. doi: 10.7150/thno.16803

Han, Y., Jia, L., Zheng, Y., and Li, W. (2018). Salivary exosomes: emerging roles in systemic disease. Int. J. Biol. Sci. 14, 633-643. doi: 10.7150/ijbs.25018

Hao, Z. C., Lu, J., Wang, S. Z., Wu, H., Zhang, Y. T., and Xu, S. G. (2017). Stem cell-derived exosomes: a promising strategy for fracture healing. Cell Prolif. 50:e12359. doi: 10.1111/cpr.12359

Hayashi, T., and Hoffman, M. P. (2017). Exosomal microrna communication between tissues during organogenesis. RNA Biol. 14, 1683-1689. doi: 10.1080/ 15476286.2017.1361098

Hoshino, A., Costa-Silva, B., Shen, T. L., Rodrigues, G., Hashimoto, A., Tesic Mark, M., et al. (2015). Tumour exosome integrins determine organotropic metastasis. Nature. 527, 329-335. doi: 10.1038/nature15756

Huang, C., Geng, J., and Jiang, S. (2017). Micrornas in regulation of osteogenic differentiation of mesenchymal stem cells. Cell Tissue Res. 368, 229-238.

Huang, C. C., Narayanan, R., Alapati, S., and Ravindran, S. (2016). Exosomes as biomimetic tools for stem cell differentiation: applications in dental pulp tissue regeneration. Biomaterials 111, 103-115. doi: 10.1016/j.biomaterials.2016.09. 029

Ikeda, E., Yagi, K., Kojima, M., Yagyuu, T., Ohshima, A., Sobajima, S., et al. (2008). Multipotent cells from the human third molar: feasibility of cell-based therapy for liver disease. Diff. Res. Biol. Divers. 76, 495-505.

Jarmalaviciute, A., Tunaitis, V., Pivoraite, U., Venalis, A., and Pivoriunas, A. (2015). Exosomes from dental pulp stem cells rescue human dopaminergic neurons from 6-hydroxy-dopamine-induced apoptosis. Cytotherapy 17, 932939. doi: 10.1016/j.jcyt.2014.07.013

Jiang, N., Xiang, L., He, L., Yang, G., Zheng, J., Wang, C., et al. (2017). Exosomes mediate epithelium-mesenchyme crosstalk in organ development. ACS Nano 11, 7736-7746. doi: 10.1021/acsnano.7b01087 
Johnsen, K. B., Gudbergsson, J. M., Skov, M. N., Pilgaard, L., Moos, T., and Duroux, M. (2014). A comprehensive overview of exosomes as drug delivery vehicles endogenous nanocarriers for targeted cancer therapy. Biochim. Biophys. Acta 1846, 75-87. doi: 10.1016/j.bbcan.2014.04.005

Kalluri, R. (2016). The biology and function of exosomes in cancer. J. Clin. Investig. 126, 1208-1215. doi: 10.1172/JCI81135

Kalra, H., Adda, C. G., Liem, M., Ang, C. S., Mechler, A., Simpson, R. J., et al. (2013). Comparative proteomics evaluation of plasma exosome isolation techniques and assessment of the stability of exosomes in normal human blood plasma. Proteomics 13, 3354-3364. doi: 10.1002/pmic.201300282

Kang, H., Lee, M. J., Park, S. J., and Lee, M. S. (2018). Lipopolysaccharidepreconditioned periodontal ligament stem cells induce $\mathrm{m} 1$ polarization of macrophages through extracellular vesicles. Int. J. Mol. Sci. 19:E3843. doi: 10. 3390/ijms19123843

Kim, H. J., and Park, J. S. (2017). Usage of human mesenchymal stem cells in cell-based therapy: advantages and disadvantages. Dev. Reprod. 21, 1-10. doi: 10.12717/DR.2017.21.1.001

Kim, J., Shin, H., and Park, J. (2017). Rna in salivary extracellular vesicles as a possible tool for systemic disease diagnosis. J. Dental Res. 96, 938-944. doi: $10.1177 / 0022034517702100$

Kowal, J., Tkach, M., and Thery, C. (2014). Biogenesis and secretion of exosomes. Curr. Opin. Cell Biol. 29, 116-125. doi: 10.1016/j.ceb.2014.05.004

Lai, R. C., Arslan, F., Lee, M. M., Sze, N. S., Choo, A., Chen, T. S., et al. (2010). Exosome secreted by msc reduces myocardial ischemia/reperfusion injury. Stem Cell Res. 4, 214-222. doi: 10.1016/j.scr.2009.12.003

Lai, R. C., Yeo, R. W., and Lim, S. K. (2015). Mesenchymal stem cell exosomes. Semin. Cell Dev. Biol. 40, 82-88.

Lai, R. C., Yeo, R. W., Tan, K. H., and Lim, S. K. (2013). Mesenchymal stem cell exosome ameliorates reperfusion injury through proteomic complementation. Regen. Med. 8, 197-209. doi: 10.2217/rme.13.4

Langer, R., and Vacanti, J. P. (1993). Tissue engineering. Science 260, 920-926.

Lee, M., Ban, J.-J., Im, W., and Kim, M. (2016). Influence of storage condition on exosome recovery. Biotechnol. Bioproc. Eng. 21, 299-304.

Li, W., Liu, Y., Zhang, P., Tang, Y., Zhou, M., Jiang, W., et al. (2018). Tissueengineered bone immobilized with human adipose stem cells-derived exosomes promotes bone regeneration. ACS Appl. Mat. Interfaces 10, 5240-5254. doi: 10.1021/acsami.7b17620

Li, Y., Wang, X., Ren, J., Wu, X., Li, G., Fan, Z., et al. (2018). Mandible exosomal ssc-mir-133b regulates tooth development in miniature swine via endogenous apoptosis. Bone Res. 6:28.

Liang, X., Ding, Y., Zhang, Y., Tse, H. F., and Lian, Q. (2014). Paracrine mechanisms of mesenchymal stem cell-based therapy: current status and perspectives. Cell Transpl. 23, 1045-1059. doi: 10.3727/096368913X667709

Liao, W., Du, Y., Zhang, C., Pan, F., Yao, Y., Zhang, T., et al. (2018). Exosomes: the next generation of endogenous nanomaterials for advanced drug delivery and therapy. Acta Biomat. 86, 1-14. doi: 10.1016/j.actbio.2018.12.045

Lizier, N. F., Kerkis, A., Gomes, C. M., Hebling, J., Oliveira, C. F., Caplan, A. I., et al. (2012). Scaling-up of dental pulp stem cells isolated from multiple niches. PLoS One 7:e39885. doi: 10.1371/journal.pone.0039885

Lousada-Fernandez, F., Rapado-Gonzalez, O., Lopez-Cedrun, J. L., Lopez-Lopez, R., Muinelo-Romay, L., and Suarez-Cunqueiro, M. M. (2018). Liquid biopsy in oral cancer. Int. J. Mol. Sci. 19:1704.

Lu, Z., Chen, Y., Dunstan, C., Roohani-Esfahani, S., and Zreiqat, H. (2017). Priming adipose stem cells with tumor necrosis factor-alpha preconditioning potentiates their exosome efficacy for bone regeneration. Tissue Eng. A 23, 1212-1220. doi: 10.1089/ten.tea.2016.0548

McKenzie, A. J., Hoshino, D., Hong, N. H., Cha, D. J., Franklin, J. L., Coffey, R. J., et al. (2016). Kras-mek signaling controls ago 2 sorting into exosomes. Cell Rep. 15, 978-987. doi: 10.1016/j.celrep.2016.03.085

Mead, B., and Tomarev, S. (2017). Bone marrow-derived mesenchymal stem cellsderived exosomes promote survival of retinal ganglion cells through mirnadependent mechanisms. Stem Cells Transl. Med. 6, 1273-1285. doi: 10.1002/ sctm.16-0428

Mendt, M., Rezvani, K., and Shpall, E. (2019). Mesenchymal stem cell-derived exosomes for clinical use. Bone Marrow Transplant. 54(Suppl. 2), 789-792. doi: 10.1038/s41409-019-0616-z

Menicanin, D., Mrozik, K. M., Wada, N., Marino, V., Shi, S., Bartold, P. M., et al. (2014). Periodontal-ligament-derived stem cells exhibit the capacity for longterm survival, self-renewal, and regeneration of multiple tissue types in vivo. Stem Cells Dev. 23, 1001-1011. doi: 10.1089/scd.2013.0490
Michael, A., Bajracharya, S. D., Yuen, P. S., Zhou, H., Star, R. A., Illei, G. G., et al. (2010). Exosomes from human saliva as a source of microrna biomarkers. Oral Dis. 16, 34-38. doi: 10.1111/j.1601-0825.2009.01604.x

Misawa, M. Y. O., Silverio Ruiz, K. G., Nociti, F. H. Jr., Albiero, M. L., Saito, M. T., Nobrega Stipp, R., et al. (2019). Periodontal ligamentderived mesenchymal stem cells modulate neutrophil responses via paracrine mechanisms. J. Periodontol. 90, 747-755. doi: 10.1002/JPER.18-0220

Mohammed, E., Khalil, E., and Sabry, D. (2018). Effect of adipose-derived stem cells and their exo as adjunctive therapy to nonsurgical periodontal treatment: a histologic and histomorphometric study in rats. Biomolecules 8:E167. doi: 10.3390/biom8040167

Nakamura, Y., Miyaki, S., Ishitobi, H., Matsuyama, S., Nakasa, T., Kamei, N., et al. (2015). Mesenchymal-stem-cell-derived exosomes accelerate skeletal muscle regeneration. FEBS Lett. 589, 1257-1265. doi: 10.1016/j.febslet.2015.03.031

Narayanan, R., Huang, C. C., and Ravindran, S. (2016). Hijacking the cellular mail: exosome mediated differentiation of mesenchymal stem cells. Stem Cells Int. 2016:3808674. doi: 10.1155/2016/3808674

Nonaka, T., and Wong, D. T. W. (2017). Saliva-exosomics in cancer: molecular characterization of cancer-derived exosomes in saliva. Enzymes 42, 125-151. doi: 10.1016/bs.enz.2017.08.002

Ohno, S., Takanashi, M., Sudo, K., Ueda, S., Ishikawa, A., Matsuyama, N., et al. (2013). Systemically injected exosomes targeted to egfr deliver antitumor microrna to breast cancer cells. Mol. Ther. 21, 185-191. doi: 10.1038/mt.2012. 180

Pan, B. T., and Johnstone, R. M. (1983). Fate of the transferrin receptor during maturation of sheep reticulocytes in vitro: selective externalization of the receptor. Cell 33, 967-978.

Papaioannou, G., Mirzamohammadi, F., and Kobayashi, T. (2014). Micrornas involved in bone formation. Cell. Mol. Life Sci. 71, 4747-4761. doi: 10.1007/ s00018-014-1700-6

Park, K. M., and Gerecht, S. (2014). Harnessing developmental processes for vascular engineering and regeneration. Development 141, 2760-2769. doi: 10. 1242/dev.102194

Phinney, D. G., and Pittenger, M. F. (2017). Concise review: Msc-derived exosomes for cell-free therapy. Stem Cells 35, 851-858. doi: 10.1002/stem.2575

Pilipchuk, S. P., Plonka, A. B., Monje, A., Taut, A. D., Lanis, A., Kang, B., et al. (2015). Tissue engineering for bone regeneration and osseointegration in the oral cavity. Dent. Mat. 31, 317-338.

Pittenger, M. F., Mackay, A. M., Beck, S. C., Jaiswal, R. K., Douglas, R., Mosca, J. D., et al. (1999). Multilineage potential of adult human mesenchymal stem cells. Science 284, 143-147. doi: 10.1126/science.284.5411.143

Qi, X., Zhang, J., Yuan, H., Xu, Z., Li, Q., Niu, X., et al. (2016). Exosomes secreted by human-induced pluripotent stem cell-derived mesenchymal stem cells repair critical-sized bone defects through enhanced angiogenesis and osteogenesis in osteoporotic rats. Int. J. Biol. Sci. 12, 836-849. doi: 10.7150/ijbs.14809

Qing, L., Chen, H., Tang, J., and Jia, X. (2018). Exosomes and their microrna cargo: new players in peripheral nerve regeneration. Neurorehabil. Neural Repair 32, 765-776. doi: 10.1177/1545968318798955

Rajan, T. S., Giacoppo, S., Diomede, F., Ballerini, P., Paolantonio, M., Marchisio, M., et al. (2016a). The secretome of periodontal ligament stem cells from ms patients protects against eae. Sci. Rep. 6:38743. doi: 10.1038/srep38743

Rajan, T. S., Giacoppo, S., Trubiani, O., Diomede, F., Piattelli, A., Bramanti, P., et al. (2016b). Conditioned medium of periodontal ligament mesenchymal stem cells exert anti-inflammatory effects in lipopolysaccharide-activated mouse motoneurons. Exp. Cell Res. 349, 152-161. doi: 10.1016/j.yexcr.2016.10.008

Rana, S., Yue, S., Stadel, D., and Zoller, M. (2012). Toward tailored exosomes: the exosomal tetraspanin web contributes to target cell selection. Int. J. Biochem. Cell Biol. 44, 1574-1584. doi: 10.1016/j.biocel.2012.06.018

Raposo, G., Nijman, H. W., Stoorvogel, W., Liejendekker, R., Harding, C. V., Melief, C. J., et al. (1996). B lymphocytes secrete antigen-presenting vesicles. J. Exp. Med. 183, 1161-1172.

Raposo, G., and Stoorvogel, W. (2013). Extracellular vesicles: exosomes, microvesicles, and friends. J. Cell Biol. 200, 373-383. doi: 10.1083/jcb. 201211138

Seo, B. M., Miura, M., Gronthos, S., Bartold, P. M., Batouli, S., Brahim, J., et al. (2004). Investigation of multipotent postnatal stem cells from human periodontal ligament. Lancet 364, 149-155.

Seo, Y., Kim, H. S., and Hong, I. S. (2019). Stem cell-derived extracellular vesicles as immunomodulatory therapeutics. Stem Cells Int. 2019:5126156. doi: 10.1155/ $2019 / 5126156$ 
Shanmuganathan, M., Vughs, J., Noseda, M., and Emanueli, C. (2018). Exosomes: basic biology and technological advancements suggesting their potential as ischemic heart disease therapeutics. Front. Physiol. 9:1159. doi: 10.3389/fphys. 2018.01159

Shi, Q., Qian, Z., Liu, D., Sun, J., Wang, X., Liu, H., et al. (2017). Gmsc-derived exosomes combined with a chitosan/silk hydrogel sponge accelerates wound healing in a diabetic rat skin defect model. Front. Physiol. 8:904. doi: 10.3389/ fphys.2017.00904

Skotland, T., Hessvik, N. P., Sandvig, K., and Llorente, A. (2019). Exosomal lipid composition and the role of ether lipids and phosphoinositides in exosome biology. J. Lipid Res. 60, 9-18. doi: 10.1194/jlr.R084343

Sun, Y., Xia, Z., Shang, Z., Sun, K., Niu, X., Qian, L., et al. (2016). Facile preparation of salivary extracellular vesicles for cancer proteomics. Sci. Rep. 6:24669. doi: 10.1038/srep24669

Tan, S. S., Yin, Y., Lee, T., Lai, R. C., Yeo, R. W., Zhang, B., et al. (2013). Therapeutic msc exosomes are derived from lipid raft microdomains in the plasma membrane. J. Extracell. Vesicles 2:22614. doi: 10.3402/jev.v2i0.22614

Tao, S. C., Yuan, T., Zhang, Y. L., Yin, W. J., Guo, S. C., and Zhang, C. Q. (2017). Exosomes derived from mir-140-5p-overexpressing human synovial mesenchymal stem cells enhance cartilage tissue regeneration and prevent osteoarthritis of the knee in a rat model. Theranostics 7, 180-195. doi: 10.7150/ thno. 17133

Tatullo, M., Marrelli, M., Shakesheff, K. M., and White, L. J. (2015). Dental pulp stem cells: function, isolation and applications in regenerative medicine. J. Tissue Eng. Regen. Med. 9, 1205-1216. doi: 10.1002/term.1899

Toh, W. S., Lai, R. C., Hui, J. H. P., and Lim, S. K. (2017). Msc exosome as a cell-free msc therapy for cartilage regeneration: implications for osteoarthritis treatment. Semin. Cell Dev. Biol. 67, 56-64. doi: 10.1016/j.semcdb.2016.11.008

Toh, W. S., Lai, R. C., Zhang, B., and Lim, S. K. (2018a). Msc exosome works through a protein-based mechanism of action. Biochem. Soc. Trans. 46, 843853. doi: 10.1042/BST20180079

Toh, W. S., Zhang, B., Lai, R. C., and Lim, S. K. (2018b). Immune regulatory targets of mesenchymal stromal cell exosomes/small extracellular vesicles in tissue regeneration. Cytotherapy 20, 1419-1426. doi: 10.1016/j.jcyt.2018.09.008

Tomasoni, S., Longaretti, L., Rota, C., Morigi, M., Conti, S., Gotti, E., et al. (2013). Transfer of growth factor receptor mrna via exosomes unravels the regenerative effect of mesenchymal stem cells. Stem Cells Dev. 22, 772-780. doi: 10.1089/scd. 2012.0266

Ullah, I., Park, J. M., Kang, Y. H., Byun, J. H., Kim, D. G., Kim, J. H., et al. (2017). Transplantation of human dental pulp-derived stem cells or differentiated neuronal cells from human dental pulp-derived stem cells identically enhances regeneration of the injured peripheral nerve. Stem Cells Dev. 26, 1247-1257. doi: $10.1089 /$ scd.2017.0068

Valadi, H., Ekstrom, K., Bossios, A., Sjostrand, M., Lee, J. J., and Lotvall, J. O. (2007). Exosome-mediated transfer of mrnas and micrornas is a novel mechanism of genetic exchange between cells. Nat Cell Biol. 9, 654-659.

van Balkom, B. W. M., Gremmels, H., Giebel, B., and Lim, S. K. (2019). Proteomic signature of mesenchymal stromal cell-derived small extracellular vesicles. Proteomics 19:e1800163. doi: 10.1002/pmic.201800163

van Niel, G., D'Angelo, G., and Raposo, G. (2018). Shedding light on the cell biology of extracellular vesicles. Nat. Rev. Mol. Cell Biol. 19, 213-228.

Villarroya-Beltri, C., Gutierrez-Vazquez, C., Sanchez-Cabo, F., Perez-Hernandez, D., Vazquez, J., Martin-Cofreces, N., et al. (2013). Sumoylated hnrnpa2b1 controls the sorting of mirnas into exosomes through binding to specific motifs. Nat. Commun. 4:2980. doi: 10.1038/ncomms3980

Wada, N., Menicanin, D., Shi, S., Bartold, P. M., and Gronthos, S. (2009). Immunomodulatory properties of human periodontal ligament stem cells. J. Cell. Physiol. 219, 667-676. doi: 10.1002/jcp.21710

Wang, Y., Chen, X., Cao, W., and Shi, Y. (2014). Plasticity of mesenchymal stem cells in immunomodulation: pathological and therapeutic implications. Nat. Immunol. 15, 1009-1016.

Wang, Z., Ding, L., Zheng, X. L., Wang, H. X., and Yan, H. M. (2014). [DCderived exosomes induce osteogenic differentiation of mesenchymal stem cells]. Zhongguo Shi Yan Xue Ye Xue Za Zhi 22, 600-604. doi: 10.7534/j.issn.10092137.2014.03.005

Watt, S. M., Gullo, F., van der Garde, M., Markeson, D., Camicia, R., Khoo, C. P., et al. (2013). The angiogenic properties of mesenchymal stem/stromal cells and their therapeutic potential. Br. Med. Bull. 108, 25-53. doi: 10.1093/bmb/ldt031
Williams, A. R., and Hare, J. M. (2011). Mesenchymal stem cells: biology, pathophysiology, translational findings, and therapeutic implications for cardiac disease. Circ. Res. 109, 923-940. doi: 10.1161/CIRCRESAHA.111. 243147

Wu, J. Y., Ji, A. L., Wang, Z. X., Qiang, G. H., Qu, Z., Wu, J. H., et al. (2018). Exosome-mimetic nanovesicles from hepatocytes promote hepatocyte proliferation in vitro and liver regeneration in vivo. Sci. Rep. 8:2471. doi: 10. 1038/s41598-018-20505-y

Xu, Q., Cui, Y., Luan, J., Zhou, X., Li, H., and Han, J. (2018). Exosomes from c2c12 myoblasts enhance osteogenic differentiation of mc3t3-e1 pre-osteoblasts by delivering mir-27a-3p. Biochem. Biophys. Res. Commun. 498, 32-37. doi: 10.1016/j.bbrc.2018.02.144

Yamada, Y., Ito, K., Nakamura, S., Ueda, M., and Nagasaka, T. (2011). Promising cell-based therapy for bone regeneration using stem cells from deciduous teeth, dental pulp, and bone marrow. Cell Transpl. 20, 1003-1013. doi: 10.3727/ 096368910X539128

Zamani, P., Fereydouni, N., Butler, A. E., Navashenaq, J. G., and Sahebkar, A. (2018). The therapeutic and diagnostic role of exosomes in cardiovascular diseases. Trends Cardiovasc. Med. 29, 313-323. doi: 10.1016/j.tcm.2018. 10.010

Zhang, B., Wang, M., Gong, A., Zhang, X., Wu, X., Zhu, Y., et al. (2015). Hucmscexosome mediated-wnt4 signaling is required for cutaneous wound healing. Stem Cells 33, 2158-2168. doi: 10.1002/stem.1771

Zhang, J., Liu, X., Li, H., Chen, C., Hu, B., Niu, X., et al. (2016). Exosomes/tricalcium phosphate combination scaffolds can enhance bone regeneration by activating the pi3k/akt signaling pathway. Stem Cell Res. Ther. 7:136. doi: 10.1186/s13287-016-0391-3

Zhang, S., Chu, W. C., Lai, R. C., Lim, S. K., Hui, J. H., and Toh, W. S. (2016). Exosomes derived from human embryonic mesenchymal stem cells promote osteochondral regeneration. Osteoarthr. Cartilage 24, 2135-2140. doi: 10.1016/ j.joca.2016.06.022

Zhang, S., Chuah, S. J., Lai, R. C., Hui, J. H. P., Lim, S. K., and Toh, W. S. (2018). Msc exosomes mediate cartilage repair by enhancing proliferation, attenuating apoptosis and modulating immune reactivity. Biomaterials 156, 16-27. doi: 10.1016/j.biomaterials.2017.11.028

Zhang, Y., Shi, S., Xu, Q., Zhang, Q., Shanti, R. M., and Le, A. D. (2018). SIS-ECM laden with GMSC-derived exosomes promote taste bud regeneration. J. Dent. Res. 98, 225-233. doi: 10.1177/0022034518804531

Zhang, Y., Chopp, M., Liu, X. S., Katakowski, M., Wang, X., Tian, X., et al. (2017). Exosomes derived from mesenchymal stromal cells promote axonal growth of cortical neurons. Mol. Neurobiol. 54, 2659-2673. doi: 10.1007/s12035-0169851-0

Zhu, W., and Liang, M. (2015). Periodontal ligament stem cells: current status, concerns, and future prospects. Stem Cells Int. 2015:972313. doi: 10.1155/2015/ 972313

Zitvogel, L., Regnault, A., Lozier, A., Wolfers, J., Flament, C., Tenza, D., et al. (1998). Eradication of established murine tumors using a novel cell-free vaccine: dendritic cell-derived exosomes. Nat. Med. 4, 594-600.

Zlotogorski-Hurvitz, A., Dekel, B. Z., Malonek, D., Yahalom, R., and Vered, M. (2019). Ftir-based spectrum of salivary exosomes coupled with computationalaided discriminating analysis in the diagnosis of oral cancer. J. Cancer Res. Clin. Oncol. 145, 685-694. doi: 10.1007/s00432-018-02827-6

Zordani, A., Pisciotta, A., Bertoni, L., Bertani, G., Vallarola, A., Giuliani, D., et al. (2019). Regenerative potential of human dental pulp stem cells in the treatment of stress urinary incontinence: in vitro and in vivo study. Cell Prolif. 52:e12675. doi: $10.1111 /$ cpr. 12675

Conflict of Interest: The authors declare that the research was conducted in the absence of any commercial or financial relationships that could be construed as a potential conflict of interest.

Copyright (c) 2020 Cooper, Ravindran, Huang and Kang. This is an open-access article distributed under the terms of the Creative Commons Attribution License (CC BY). The use, distribution or reproduction in other forums is permitted, provided the original author(s) and the copyright owner(s) are credited and that the original publication in this journal is cited, in accordance with accepted academic practice. No use, distribution or reproduction is permitted which does not comply with these terms. 